Natural Hazards and Earth System Sciences (2003) 3: 197-201

(C) European Geosciences Union 2003

Natural Hazards and Earth System Sciences

\title{
Exalting in atmospheric tides as earthquake precursor
}

\author{
P. F. Biagi ${ }^{1}$, R. Piccolo ${ }^{1}$, V. Capozzi ${ }^{2}$, A. Ermini ${ }^{3}$, S. Martellucci ${ }^{3,4}$, and C. Bellecci ${ }^{3,4}$ \\ ${ }^{1}$ Department of Physics-INFM, University of Bari, Via Amendola, 173, 70126 Bari, Italy \\ ${ }^{2}$ Medicin Faculty-INFM, University of Foggia, Via L. Pinto, 71100 Foggia, Italy \\ ${ }^{3}$ Department of Physics and Energy Science and Technology, University of Roma "Tor Vergata”, Via di Tor Vergata, 00133 \\ Rome, Italy \\ ${ }^{4}$ INFM, University of Roma "Tor Vergata", Italy
}

Received: 13 May 2002 - Revised: 20 June 2002 - Accepted: 21 June 2002

\begin{abstract}
During February-March 1998, we observed a significant increase $(6-8 \mathrm{~dB})$ in the electric field of the CZE ( $f=270 \mathrm{kHz}$, Czech Republic) broadcasting station at a site named AS and located in central Italy. On 13 March 1998 an earthquake $(M=5.2)$ occurred in Slovenia, starting a strong seismic crisis $(M=6.0$ on 12 April, $M=5.1$ on 6 May). The distances of the epicentres from the radio receiver were over $400 \mathrm{~km}$, but the epicentres are located in a zone that is in the middle of the CZE-AS path. Previously, we advanced the hypothesis that the increase of radio-signal electric field detected could be a precursor of the Slovenia seismic crisis. At the purpose to precise the connection between this radio anomaly and the preparatory phase of the earthquakes, we analysed in detail the data collected. At first we carried out a FFT on the data and clear harmonic components with period $0.5 \mathrm{~d}, 1 \mathrm{~d}, 14 \mathrm{~d}, 28 \mathrm{~d}$ and $365 \mathrm{~d}$ were revealed. These periods are characteristic of gravity tides and therefore, it seems reasonable to consider the previous harmonics in the electric field of the radio signal as components related to tides of the atmosphere, assuming as tides of the atmosphere only the linear action of gravity tides on atmosphere. Then, we carried out band pass filters on our data and we discovered that the February-March 1998 increase is mainly related to an exalting of the harmonics with period $0.5 \mathrm{~d}, 1 \mathrm{~d}$, $14 \mathrm{~d}$ and $28 \mathrm{~d}$, i.e. of the (semi)monthly and (semi)diurnal atmospheric tidal components in the radio signal. Supposing that this exalting is produced by a corresponding exalting of the atmospheric tides we propose a model able to explain the production of such an effect during the preparatory phase of the Slovenia earthquakes.
\end{abstract}

\section{Introduction}

Among the radio signals, low frequency (LF) radio signals lie in the band $30-300 \mathrm{kHz}$. LF radio wave reception is characterised mainly by sky-wave propagation modes (Knight,

Correspondence to: P. F. Biagi (biagi@ fisica.uniba.it)
1973). The sky-wave signal is variable between day and night and between winter and summer in consequence of the different state of the lower ionosphere. In 1996 we designed and built a receiver able to measure the electric field strength of three LF radio signals at field sites where the noise is very low. We selected the LF broadcasting stations MCO (France), CZE (Czech Republic) and CLT (Italy). The monitoring equipment is detailed in Bella et al. (1989). At the end of 1996 we put into operation the receiver at a site in central Italy named AS. In Fig. 1, the location of the transmitters and of the receiver is shown. The radiated power and frequency of the broadcasting stations, together with the distance from the receiver, is also indicated in Fig. 1. Some possible seismic-disturbances in these radio signals have been presented previously (Bella et al., 1998; Biagi, 1999; Biagi et al., 2001). In particular, the whole of the data recorded since January 1997 was examined (Biagi et al., 2001). Firstly, a 7-day high pass filter was applied to the raw data to remove high frequency components and then the long term smoothed trend of the filtered data was determined by fitting a 9th order polynomial. Next, a working file was created as the difference between the filtered set and its polynomial fit. Lastly the standard deviation $\sigma$ over the entire work data set was calculated. So, an anomalous increase over the $3 \sigma$ level on the CZE trend during February-March 1998 (Fig. 2) was discovered. This anomaly did not appear on MCO and CLT trends and might have been a precursor of the strong seismic sequence ( $M=5.1-6.0)$ that occurred on March-May 1998 in Slovenia at a location over $400 \mathrm{~km}$ from the receiver, but lying in the middle of the transmitter-receiver path (Biagi et al., 2001). The time occurrence of the sequence is indicated in Fig. 2 and the location of epicentres is shown in Fig. 1.

\section{Analysis}

At the purpose of improving the definition of the radio anomaly described in Sect. 1 we carried out a spectral analysis on the CZE data. We examined the original data, the 
Table 1. Parameters of some tidal constituent of gravity

\begin{tabular}{cccc}
\hline Name & $\begin{array}{c}\text { Period } \\
\text { (mean solar days) }\end{array}$ & $\begin{array}{c}\text { Relative Coefficient } \\
(\mathrm{M} 2=1.0000)\end{array}$ & Origin \\
\hline $\mathrm{S}_{a}$ & 364.96 & 0.0127 & Solar annual \\
$\mathrm{S}_{s a}$ & 182.70 & 0.0802 & Solar semi-annual \\
$\mathrm{M}_{m}$ & 27.55 & 0.0909 & Lunar monthly \\
$\mathrm{M}_{f}$ & 13.66 & 0.1723 & Lunar semi-monthly \\
$\mathrm{K}_{l}$ & 0.997 & 0.3990 & Principal lunar \\
$\mathrm{K}_{l}$ & 0.997 & 0.1852 & Principal solar \\
$\mathrm{M}_{2}$ & 0.518 & 1.0000 & Principal lunar \\
$\mathrm{S}_{2}$ & 0.500 & 0.4652 & Principal solar \\
\hline
\end{tabular}

low pass filter ( 8 days) data and the band pass filter (8-120 days) data. These data sets are shown in Fig. 3a. The relative spectra are given in Fig. 3b. In the first case the main periods are 1 day and 0.5 day with their harmonics; in the second case the main period is 365 days with its harmonics. In the last case several components exist; among them components with period 28 days and 14 days. We carried out the spectral analysis also on the MCO and CLT data and the result we obtained was the same. The different state of the lower ionosphere during day and night and during summer and winter could justify the $1 \mathrm{~d}$ and $365 \mathrm{~d}$ periods, but it cannot do the same for the other periods. On the contrary, all the previous periods are characteristic of the gravitational tides. The period and other characteristics of some tidal constituents of gravitational field are indicated in Table 1 . We can exclude that the gravitational tides act on transmitters and receiver or directly on the radio signals, that are electromagnetic waves. On the contrary an action on the radio signals propagation medium exists, i.e. the atmospheric tides. So, from the previous analysis it seems that the atmospheric tides affect the propagation of LF radio signals. Then we continued the analysis and we applied on the CZE original data narrow band pass filters centred on the periods of the main (semi)monthly and (semi)diurnal tidal components. The result we obtained is shown in Fig. 4. From Fig. 4 an exalting of the radio signal tidal harmonics appears to be correspondent with the radio anomaly we described in Sect. 1. Such a correspondence does not appear using band pass filters that do not include any tidal harmonic. The trend at the bottom of Fig. 4 (band pass 22-24d; no tidal constituents) is an example of such a correspondence lacking. So, this analysis has shown that the radio anomaly we are studying is formed by an exalting of its main (semi)diurnal and (semi)monthly tidal $(0.5 \mathrm{~d}, 1 \mathrm{~d}$, $14 \mathrm{~d}$ and 28d) harmonics.

\section{Discussion}

The atmosphere is characterized by oscillations related to several causes (Molchanov et al., 2001). As it concerns the action of gravity tides the atmosphere, due to its complex nature, acts like a non linear system. So, not only the oscilla-

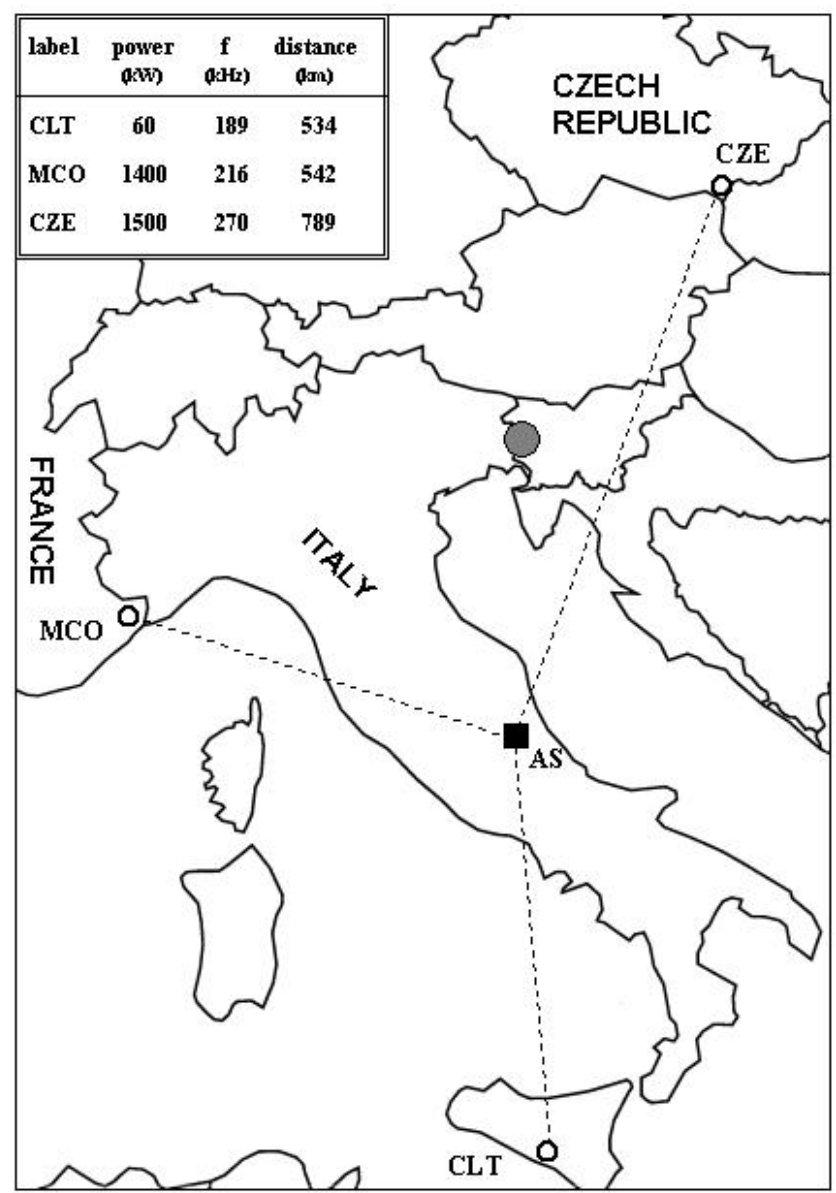

Fig. 1. Map showing the radio broadcasting stations (CLT, MCO, $\mathrm{CZE}$ ) and the measurement site (AS). The radiated power and frequency of the broadcasting stations, together with the distance from the receiver, are indicated in the panel. The grey circle shows roughly the location of the Slovenia seismic crisis.

tions with the same periods of gravity tides will exist (linear response), but also oscillations with several different periods (non linear response) will be generated. In this study we have taken into consideration only the linear response, so we considered as atmospheric tides only those atmospheric oscillations with the same periods of the gravity tides. Now let us consider the effect of these atmospheric tides on the propagation of LF radio signals. We assume that such a tides can produce variation in the ionospheric reflection coefficient $R$ (CCIR, 1990a, b), that is the main parameter that control the LF sky wave propagation. Even if the precise interaction is not well understood, we suppose that the atmospheric tides affect the collisions between electrons and neutral particles in the lower ionosphere, producing different periodic variations in the collision frequency. So, similar variations in $R$ parameter could be produced and, therefore, the electric field intensity of LF radio waves at a receiver will change accordingly. Of course, other causes different from gravity tides produce periodic modifications in lower ionosphere, at first the solar radiation that produces modifications mainly from 


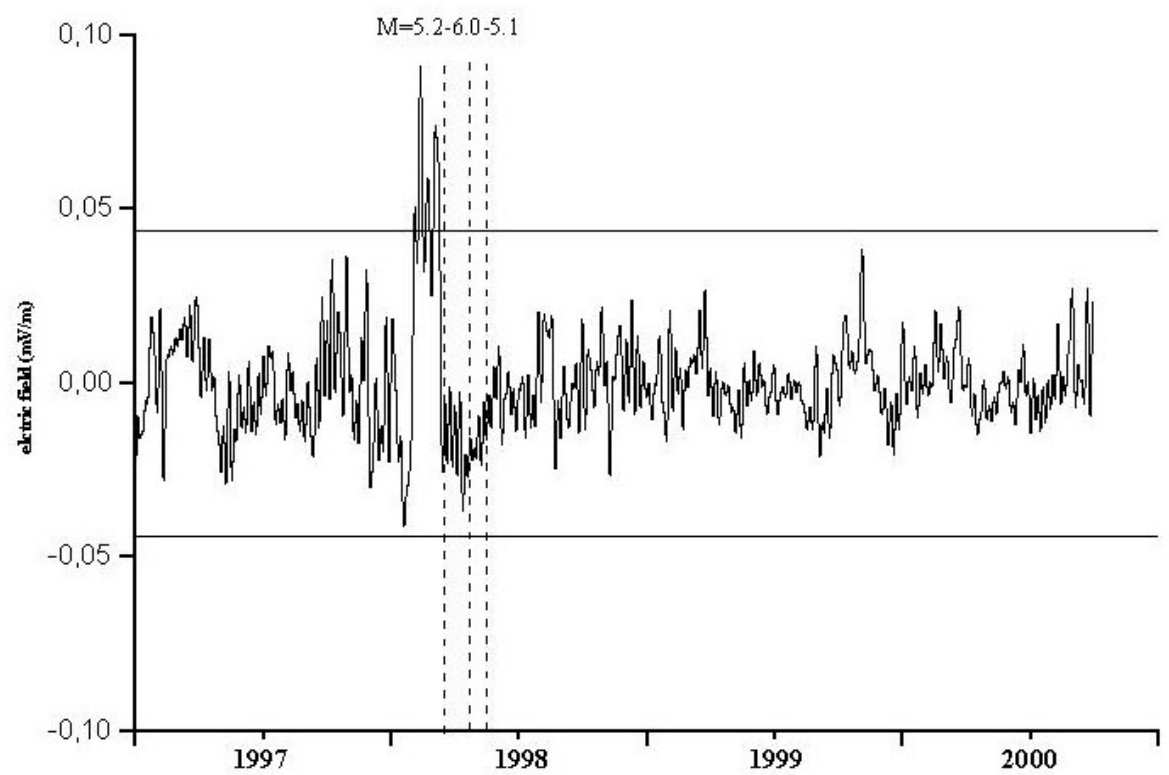

Fig. 2. Difference between the filtered data and smoothed trends of the CZE radio-signals at the AS site from 1 January 1997 to 26 September 2000. The horizontal lines represent the $\pm 3 s$ level (where $s$ is the standard deviation). The vertical dashed lines represent the occurrence of the main earthquakes ( $M=5.2,13$ March 1998; $M=6.0,12$ April 1998; $M=5.1,6$ May 1998) in the Slovenia region.
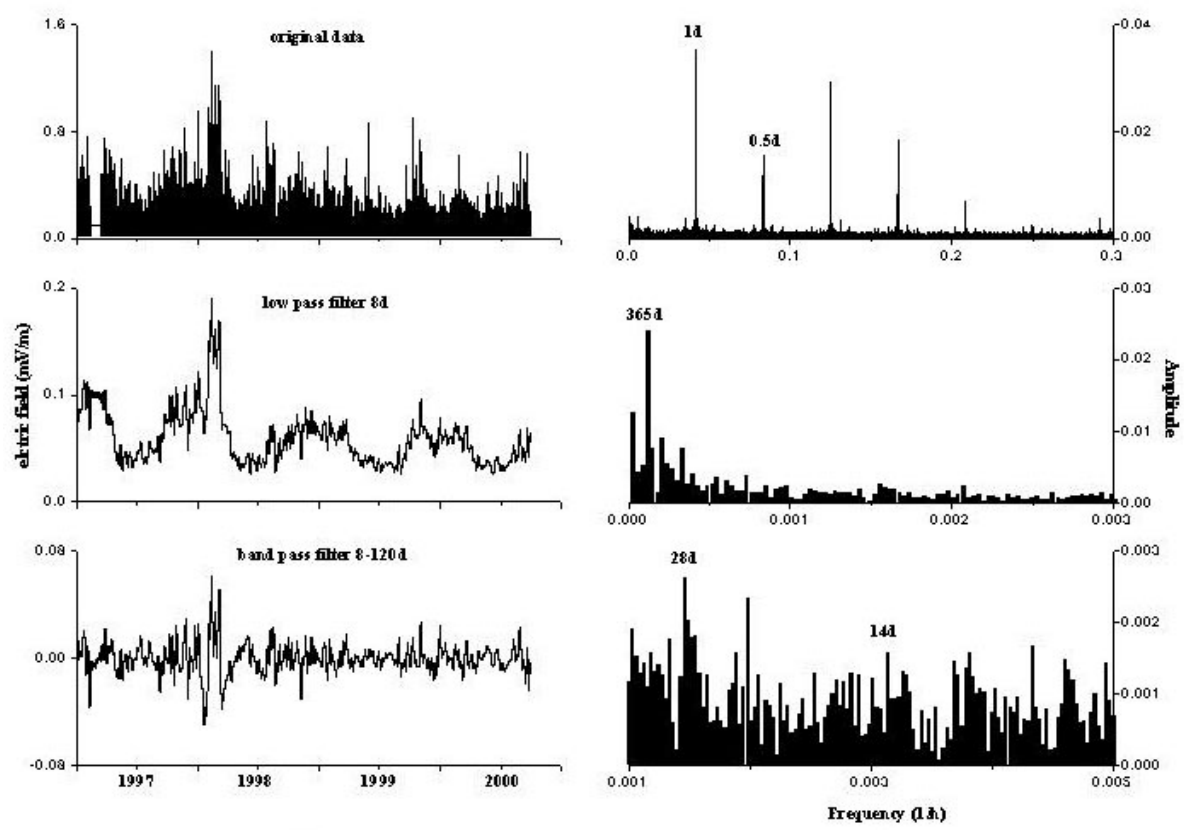

a]

b]

Fig. 3. (a) From the top, original time-series, low pass filter (8d) and band pass filter (8-120d) of the CZE radio-signals from 1 January 1997 to 26 September 2000. (b) Spectral analysis using $2 n$ values of continuous data sets obtained from the corresponding data indicated in (a). In each spectrum the main harmonics are indicated.

day to night and from winter and summer. In such a case its effect on the propagation of LF radio signals will be superimposed with the atmospheric tides effect. The next step was to examine the exalting of the $0.5 \mathrm{~d}, 1 \mathrm{~d}, 14 \mathrm{~d}$ and $28 \mathrm{~d}$ harmonics in CZE radio signal during February-March 1998. At first let us assume that the amplitude increase in these components of the radio-signal is produced by an exalting of the same period atmospheric tides. Then, because the radio anomaly we are studying was observed only on the CZE signal, it means that this effect occurred only in the north-eastern part of the 


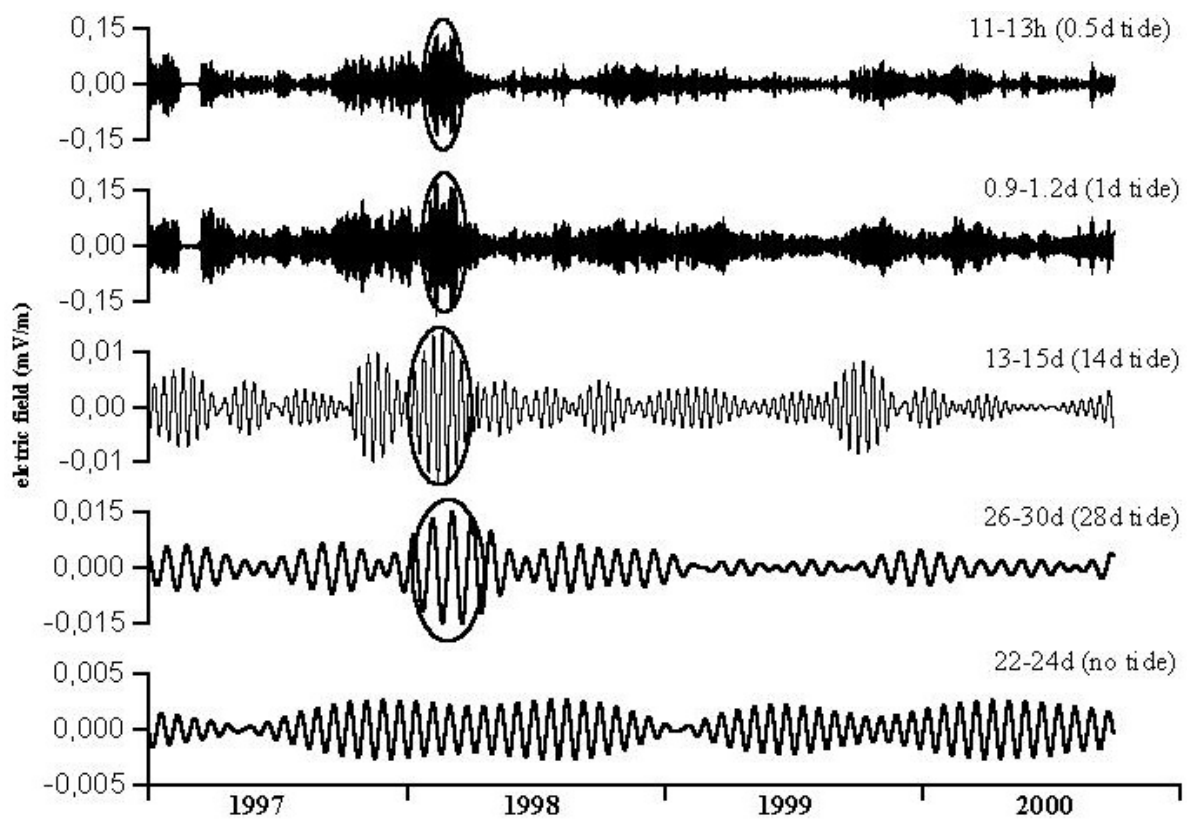

Fig. 4. From the top, band pass filters of the CZE radio-signal from 1 January 1997 to 26 September in the bands: $11-13 \mathrm{~h}, 0.9-1.2 \mathrm{~d}$, 13-15d, 26-30d and 22-24d. The first four bands are centred on the tidal constituents with period: $0.5 \mathrm{~d}, 1 \mathrm{~d}, 14 \mathrm{~d}$ and $28 \mathrm{~d}$; the last one is not centred on any tidal constituent. The exalting in the first four trends in correspondence with the radio anomaly shown in Fig. 2 is encircled.

map in Fig. 1. So, we can try to justify how the preparatory phase of Slovenia earthquakes could have produced such a local exalting of the atmospheric tides. A possible explanation is as it follows. Let us assume that the preparatory phase of the Slovenia seismic crisis produced a diffusion of fluids in a wide area around the future epicentres. This assumption seems to be reasonable; in fact emanation of gases $(\mathrm{Rn}, \mathrm{He}, \mathrm{Ar} . .$.$) and increase in the flow rate of springs or in$ water level of wells were observed many times before earthquakes (Hauksson, 1981; King et al., 1981; King, 1984/1985, Barsukov et al., 1984/1985; Roeloffs, 1988; Roeloffs et al., 1997; Bella et al., 1995; Biagi et al., 1999). As a consequence of this fluid diffusion the ground density in the zone decreased. Therefore, a local decrease of the earth gravitational field $g$ occurred. The $g$ variation can be estimated by the relation (Telford et al., 1990):

$\Delta g=2 \cdot \pi \cdot G \cdot \rho_{r} \cdot d$,

where $\Delta g$ is the gravity variation, $G$ the universal gravitational constant, $\rho_{r}$ the density variation in a surface slab of thickness $d$. As a consequence of this $g$ decrease, the lower atmosphere overlooking the perturbed zone expands and so a decrease of its density happens. In consequence of this density decrease the local atmospheric tides can enlarge.

As an example, let us consider a realistic model with a $\rho_{r}=0.1 \mathrm{~g} / \mathrm{cm}^{3}$ density decrease in a surface slab of some hundred metres thickness. On the basis Eq. (1) we obtain a $g$ decrease of some $10^{-5} \mathrm{~m} / \mathrm{s}^{2}$. Note that the range of gravitational tides is some $10^{-6} \mathrm{~m} / \mathrm{s}^{2}$, that is ten times less than the $g$ variation.

\section{Conclusions}

Harmonics with period $0.5 \mathrm{~d}, 1 \mathrm{~d}, 14 \mathrm{~d}, 28 \mathrm{~d}$ and $365 \mathrm{~d}$ in the spectral content of three LF radio signals recorded during a period of four years at a site located in central Italy indicates that the atmospheric tides (linear action of gravity tides on atmosphere) are one of the factors affecting the propagation of LF radio waves. A preseimic exalting of the atmospheric tides could justify the anomalous increase on the trend of one of the previous radio signals we observed during February-March 1998. We advance the hypothesis that such an exalting was caused by a local decrease of the gravitational field as a consequence of the preseismic fluid diffusion in a wide area around the future epicentres. In conclusion, the model we propose makes use of gravity changes to justify the preseismic anomaly observed in the LF radio signal. Recently, anomalies in VLF radio signals observed in Japan were explained in a similar way (Hayakawa and Sato, 1994; Hayakawa et al., 1996; Molchanov and Hayakawa, 1998).

\section{References}

Barsukov, V. L., Varshal, G. M., and Zamokina, N. S.: Recent results of hydrogeochemical studies for earthquake prediction in USSR, Pure Appl. Geophys., 122, 143-156, 1984/1985.

Bella, F., Bella, R., Biagi, P. F., Della Monica, G., Ermini, A., Manjgaladze, P., Sgrigna, V., and Zilpimiani, D.: A digital recording system of electromagnetic emissions, Nuovo Cimento, 12C, 251-259, 1989.

Bella, F., Biagi, P. F., Caputo, M., Cozzi, E., Della Monica, G., Ermini, A., Plastino, W., Sgrigna, V., and Zilpimiani, D.: Helium content in thermal waters in the Caucasus from 1985 to 1991 and 
correlations with the seismic activity, Tectonophysics, 246, 263278, 1995.

Bella, F., Biagi, P. F., Caputo, M., Cozzi, E., Della Monica, G., Ermini, A., Plastino, W., and Sgrigna, V.: Field strength variations of LF radio waves prior to earthquakes in Central Italy, Phys. Earth Planet. Int., 105, 279-286, 1998.

Biagi, P. F., Bella, F., Cozzi, E., Ermini, A., Scandone, R., Khatkevich, Y. M., Gordeev, E. I., Kingsley, S. P., Anderson, C. W., and Derlien, P. J.: Groundwater Argon content on the occasion of strong earthquakes in a seismogenetic area of Kamchatka (Russia), Il Nuovo Cimento C, 22, 503-508, 1999.

Biagi, P. F.: Seismic Effects on LF Radiowaves, in: Atmospheric and Ionospheric Electromagnetic Phenomena Associated with Earthquakes, (Ed) Hayakawa, M., TERRAPUB, Tokyo, 535542, 1999.

Biagi, P. F., Piccolo, R., Ermini, A., Martellucci, S., Bellecci, C., Hayakawa, M., Capozzi, V., and Kingsley, S. P.: Possible earthquake precursors revealed by LF radio signals, NHESS, 1 , No. $1-2,2001$

CCIR: Radiometeorogical data, Rep. 563-4, 105-148, 1990a.

CCIR: Sky-wave propagation and circuit performance at frequencies between about $30 \mathrm{kHz}$ and $500 \mathrm{kHz}$, Rep. 265-7, 212-229, $1990 \mathrm{~b}$

Hayakawa, M. and Sato, H.: Ionospheric perturbations associated with earthquakes, as detected by subionospheric VLF propagation, in: Electromagnetic Phenomena Related to Earthquake Prediction, (Eds) Hayakawa, M. and Fujinawa, Y., TERRAPUB, Tokyo, 391-397, 1994.
Hayakawa, M., Molchanov, O. A., Ondoh, T., and Kawai, E. Anomalies in the subionospheric VLF signals for the 1995 Hyogo-ken earthquake, J. Phys. Earth, 44, 413-418, 1996.

Hauksson, E.: Radon content of groundwater as an earthquake precursor: evaluation of wordwide data and physical basis, J. Geophys. Res., 86, 9397-9401, 1981.

King, C. Y., Evans, W. C., Presser, T., and Husk, R. H.: Anomalous chemical changes in well waters and possible relation to earthquakes, Geophys. Res. Lett., 8, 425-428, 1981.

King, C.-Y. (Ed): Earthquake hydrology and chemistry, Special Issue, Pure Appl. Geophys., 122, 141-618, 1984/1985.

Knight, P.: MF propagation: a wave-hop method for ionospheric field-strength prediction, BBC Eng., 10, 22-34, 1973.

Molchanov, O. A. and Hayakawa, M.: Subionospheric VLF signal perturbations possibly related to earthquakes, J. Geophys. Res., 103, 17 489-17 504, 1998.

Molchanov, O. A., Hayakawa M., and Miyaki, K.: VLF/LF sounding of lower ionosphere to study the role of atmospheric oscillations in the lithosphere-ionosphere coupling, Adv. Polar Upper Atmos. Res., 15, 146-158, 2001.

Roeloffs, E. A.: Hydrologic precursors to earthquakes: A review, Pure Appl. Geophys., 126, 177-209, 1988.

Roeloffs, E. A. and Quilty, E.: Case 21-water level and strain changes proceding and following the 4 August 1985 Kettleman Hills, California, earthquake, Pure Appl. Geophys., 149, 21-60, 1997.

Telford, W. M., Geldart, L. P., and Sheriff, R. E.: Applied Geophysics, Cambridge University Press, 1990. 